\title{
Proyección del estudiante de Patología y Clínica Estomatológica desde el ámbito del claustro de la Facultad al Hospital Público: Registro retrospectivo de las patologías observadas
}

Mercado $M L *$, Rom $M^{* *}$, Micinquevich $\mathrm{S}^{* * *}$, Casariego $\mathrm{Z}^{* * * *}$

\section{RESUMEN}

Estableciendo las bases del proceso Enseñanza/Aprendizaje de la Estomatología para alumnos del último nivel de la currícula de Odontología, y en especial de la Asignatura Patología y Clínica Estomatológica, se ha diseñado este estudio. El mismo se ha llevado a cabo teniendo en cuenta los siguientes Objetivos: 1) La observación del desempeño de los estudiantes en un hospital público, en donde han realizado su trabajo en terreno. 2) Un relevamiento de las lesiones y enfermedades que se han presentado con la observación y guía por el personal docente y la resolución de los casos clínicos.

Material y Métodos: los alumnos del nivel quinto de la Asignatura de Patología y Clínica Estomatológica de la Facultad de Odontología de la Plata, año tras año han sido citados, por comisiones pequeñas (no más de 7 alumnos por cada una) con el fin de observar y participar de la tarea hospitalaria que realiza un equipo docente de Estomatología dentro del funcionamiento del Servicio de Odontología de un Hospital de Agudos. Se tomaron los datos correspondientes a un tiempo estimado en horas de 1.800 horas paciente/docente-alumno. De 495 pacientes fueron evaluados los datos de edad, sexo, ocupación, estado civil, hábitos y factores ambientales. Se registraron los datos correspondientes a las lesiones y enfermedades y se realizó el análisis estadístico de los mismos.

Resultados: 1) Los alumnos demostraron interés y entusiasmo al concurrir a la Unidad Hospitalaria. Este resultado se midió por la cantidad de alumnos que solicitaron poder concurrir al Hospital fuera de las horas de clases en forma voluntaria: $40 \%$. 2) Del estudio del relevo de datos, las micosis superficiales fueron las más prevalentes seguidas por aftas, líquenes atípicos y carcinomas a células escamosas.

Conclusiones: 1) Se observa que el Hospital docente asistencial pareciera brindar al mecanismo de Enseñanza/Aprendizaje, la posibilidad de insertar al alumno en la realidad de la salud pública del medio ambiente al cual pertenece. 2) Las demás especialidades, integradas al Servicio de Odontología/Estomatología, permitirían una acción en conjunto del equipo sanitario, por un fenómeno de retroalimentación. 3) Coincidiendo con otros datos de diversos Servicios Asistenciales y otras Facultades del país, la Candidiasis oral resultó la lesión más frecuentemente observada. 4) La incidencia de Cáncer bucal nos lleva a insistir sobre su diagnóstico temprano y la importancia del reconocimiento de las enfermedades precancerosa y establecer estrategias contra el hábito del tabaquismo, en nuestro medio de referencia.

Palabras claves: Enseñanza/aprendizaje, fenómeno de retroalimentación, inserción.

\section{ABSTRACT}

This study was designed in order to establish basement of Teaching/Learning process in Stomatology, for students of the last step of Curricula in Odontology and, very specially for Patology and Stomatological Clinic' 
attenden. Objectives were: 1) Observation of students behaviour in a public hospital, were it takes place, their "work in situ". 2) To stand out diagnosticated lesions and diseases with the guide of experimental teachers and, resolution of clinic cases.

Material and methods: Students of the last level of Patology and Clinical Stomatology Asignature, of the Odontology Faculty of La Plata City, year by year were sending in small groups (no more than seven students each), to a Public Hospital, to Odontology Unit, to participate in an equipment composed by Docents of the Faculty that works in this hospitalry Service and in relationship with other medical units of the same Hospital. For this study, data was assesed taking 1.800 hours/patient. 495 patients were evaluated and sex, age, civil state, ocupation, habits and factors of the enviroment were tabulated. Screaning of lesions and diseases were made and an statistic analyse was performed.

Results: 1) Students demostrated, most of the time, really interesting and entusiassm. This result was calculated in a frequence of $40 \%$ of them, who wanted to state longer overthere and to come back, out of their own study classes and obligations. 2) From lesions and diseases data study, superficial mycosis was the most prevalent disease that it was founded, followed by major afthous and carcinomas of squamous cells.

Conclusions: 1) Its was observed that Assistant/Docent/ Hospital educational method woul offer to Teaching/ Learning mechanism, the possibility to insert the students inside their real public health medium. 2) Other medical specialities integrated with Stomatology seems to allow a common sanitary equipment by a retronutritionary phenomem. 3) In agreement with other data of asistant services and other faculties of our country, Oral Candidiasis was the most observed disease. 4) Incidence of Oral cancer advices us to insist about its early diagnosis, the importance of pre-malignant lesions recognition and to improve strategies againts nicotinism, in our enviroment.

Key words: Teaching/Learning, retronutritionary phenomem, insertion.

Aceptado para publicación: Noviembre 2005.

* Jefe de Trabajos Prácticos.Cátedra de Patología Estomatológica, Facultad de Odontología, Universidad Nacional de La Plata.

** Profesora Adjunta, Cátedra de Patología Estomatológica, Facultad de Odontología, Universidad Nacional de La Plata.

*** Profesora Adjunta, Cátedra de Patología Estomatológica, Facultad de Odontología, Universidad Nacional de La Plata.

**** Profesora Académica de Patología Estomatológica, Facultad de Odontología. Universidad Nacional de La Plata. Estomatóloga Docente en el Servicio de Infectología, Hospital de Agudos Juan A Fernández, Buenos Aires.

Mercado ML, Rom M, Micinquevich S, Casariego Z. Proyección del estudiante de Patología y Clínica Estomatológica desde el ámbito del claustro de la Facultad al Hospital Público: Registro retrospectivo de las patologías observadas. Av. Odontoestomatol 2006; 22 (5): 279-285.

\section{INTRODUCCIÓN}

El proceso enseñanza/aprendizaje de la Patología y Clínica Estomatológica tiene por objetivo general, el desenvolvimiento progresivo de todos aquellos elementos, cognoscitivos y prácticos, que van a servir al cursante como herramientas auxiliares para transmitir el conocimiento de las lesiones y enfermedades que afectan al Sistema estomatognático (mucosa bucal y órganos anexos). A través del cumplimiento de este propósito, se crea así un futuro profesional con un espíritu crítico capaz de llegar a un diagnóstico certero y que pueda efectuar el tratamiento indicado o la derivación oportuna, temprana y correcta, en caso que sea necesario. $(1,2)$

La exploración clínica como método básico dentro de la Semiología, la observación, la utilización de 
Mercado ML, Rom M, Micinquevich S, Casariego Z Proyección del estudiante de Patología y Clínica Estomatológica desde el ámbito del claustro de la Facultad al Hospital Público

todos los métodos auxiliares con los cuales hoy se cuenta, y el estudio de las imágenes por otro, juntamente con el juicio crítico, serán los pilares sobre los cuales se deberá su formación. (3)

Las necesidades educativas del alumno de pregrado tendrán en cuenta un avance gradual de los contenidos y las destrezas que, irán de lo más fácil a lo más complejo. O sea, de considerar primero las lesiones, luego las enfermedades y por último los síndromes. Las necesidades científicas irán surgiendo de la misma manera y las necesidades sociales se verán cumplimentadas al agudizar su interés por los problemas de salud pública y la población que sólo se ven en el Hospital público.

No solamente en los seminarios resueltos en la Facultad si no también la Unidad Hospitalaria se insistirá en el tema de prevención, sobre las enfermedades de transmisión sexual, el tabaquismo, el alcoholismo, la droga y la prevención de lesiones premalignas y el cáncer oral.

Por ello, nuestros objetivos educacionales en el ámbito de la unidad hospitalaria son:

- Los alumnos, al entrar en contacto con los pacientes, se integrarán al sistema de Salud Pública, adquiriendo así los aspectos de; evidencia científica, los aspectos epidemiológicos y los problemas socioeconómicos de la Salud Pública.

- Incursionar en una semiología y terapéutica global, patrimonio de varios servicios médicos diferentes en relación con la estomatología.

- Valorar el intercambio interdisciplinario y el rol que juega un odontólogo en este campo.

- Sentar las bases de la estomatología como un campo poco explorado, tierra de muchos y de nadie.

- Realizar un rastreo sobre todas las lesiones y enfermedades que han sido registradas por los docentes a cargo de la Unidad Hospitalaria en 1.800 horas/paciente, de 495 pacientes, y que han sido compartidos en la tarea docente asistencial por los alumnos cursantes de los últimos niveles de: Patología y Clínica Estomatológica de la Facultad de Odontología de la Plata, asignatura de Patología y Clínica Estomatológica.

\section{MATERIAL Y MÉTODOS}

Los alumnos de la Asignatura de Patología y Clínica Estomatológica de los niveles cuarto y quinto, fueron citados en grupos de siete alumnos, para concurrir al Servicio de Odontología del Hospital de Agudos R. Gutiérrez de la ciudad de La Plata. En ese ámbito, la Asignatura ha desarrollado su Unidad Hospitalaria. Los alumnos en forma progresiva han ido efectuando todo lo que incumbe al estudio semiológico del paciente, confeccionando historias clínicas, y acompañando a los docentes en el manejo hospitalario del paciente, analizando los resultados de los análisis clínicos, de las citologías y biopsias, de las radiografías y tomografías, etc. También han intervenido en las interconsultas que se han realizado con los demás servicios y en la visita a pacientes internados en las salas del Hospital. Al finalizar cada concurrencia se ha efectuado una evaluación oral del tipo coloquial para obtener los resultados de esta práctica.

Para el presente estudio se ha tomado el período comprendido entre los años 1994 y 2003 durante el cual han concurrido 2.500 alumnos.

Las lesiones, enfermedades y síndromes que presentaron 495 pacientes durante 1.800 horas de atención docencia-asistencial, han sido agrupadas para su mejor registro, de la siguiente manera:

\section{Infecciones de la mucosa bucal:}

a) Enfermedades bacterianas (TBC, sífilis, impétigo, sinusitis).

b) Micóticas: superficiales (candidiasis eritematosa, candidiasis pseudomembranosa, candidiasis subplaca, candidiasis esofágica) y profundas (histoplasmosis).

c) Virosis (VHS, gingivoestomatitis herpética, HPV (condilomas-verrugas vulgares) HIV y paramixovirus).

d) Parasitosis.

2. Lesiones de enfermedades Inmunológicas: Aftas, Pénfigo Vulgar, Liquen típico, Lupus sistémico, Esclerodermia, Alergias.

3. Alteraciones de la ATM: síndrome dolor-disfunción, latero desviación, Trismus.

4. Patología de las Glándulas Salivales: Quistes, Malformaciones, Alteraciones funcionales, Inflamatorias). 
5. Patología de las piezas dentarias: Pulpitis, Procesos Periapicales, Fístulas, Dientes retenidos).

6. Lesiones Gingivales y periodontales: Hiperplasia gingival, Gingivitis marginal: aguda y crónica, Periodontitis.

7. Seudotumores de la mucosa bucal: Pseudopapiloma diapneusico, hiperplasias, granuloma piógeno, granuloma gravídico, granuloma gigantocelular periférico, torus.

8. Tumores benignos: Hemangiopericitoma, papiloma, hemangioma, neurinoma, lipoma, osteofibroma, cementoma.

9. Malformaciones de los tejidos blandos: Puntos de Fordyce, lengua escrotal.

10. Alteraciones de la Pigmentación: Melanosis, vitíligo.

11. Transtornos bucales de origen neurológico: Glosodinia, neuralgia, síndrome de ardor-quemazón.

12. Alteraciones Hematológicas: Púrpura, varicosidades, úlcera eosinófila.

13. Lesiones Precancerosas: Leucoplasia, liquen atípico, eritroplasia, úlcera traumática.

14. Tumores Malignos: Carcinoma de células escamosas, linfoma lo Hodking, carcinoma basocelular, G. letal de la línea media y

15. Enfermedades Sistémicas: Bulimia, hipotiroidismo, diabetes, hipertensión, colesterolemia, cardiopatías.

Se realizaron pedidos de análisis clínicos, estudios anatomopatológicos, inmunohistoquímicos y de PCR. Para ello se enviaron muestras provenientes de citologías exfoliativas y biopsias las cuales también se consideraron en el estudio estadístico. El gabinete de radiología ha sido utilizado en forma frecuente.

La Unidad ha documentado los casos clínicos mediante fotografías clínicas.

\section{RESULTADOS}

\section{a) De los alumnos}

La evaluación del tipo coloquial en todos los grupos ha dado resultados alentadores. La evaluación se clasificó en: a) positiva; b) dudosa; c) negativa, habiendo sido positiva en un $100 \%$.

\section{b) De los casos clínicos}

La distribución por grupos etarios nos muestra una mayor incidencia de las patologías entre los 60 a 69 años, siendo la edad media de presentación de los pacientes de 46 años, con extremos entre 2 y 91 años. La relación entre sexos fue: mujeres, 306 (62\%) y hombres, 189(38\%), considerando las ocupaciones de los pacientes: las amas de casa fueron las más afectadas, 121 (25,8\%); los jubilados, 98 (20,9\%); los empleados, 78 (13,64\%); estudiantes, $64(13,64 \%)$; cuenta propistas, 40 (8,53\%); profesionales, $36(7,67 \%)$ y desocupados, $32(6,82 \%)$. Figuras $1-3$.

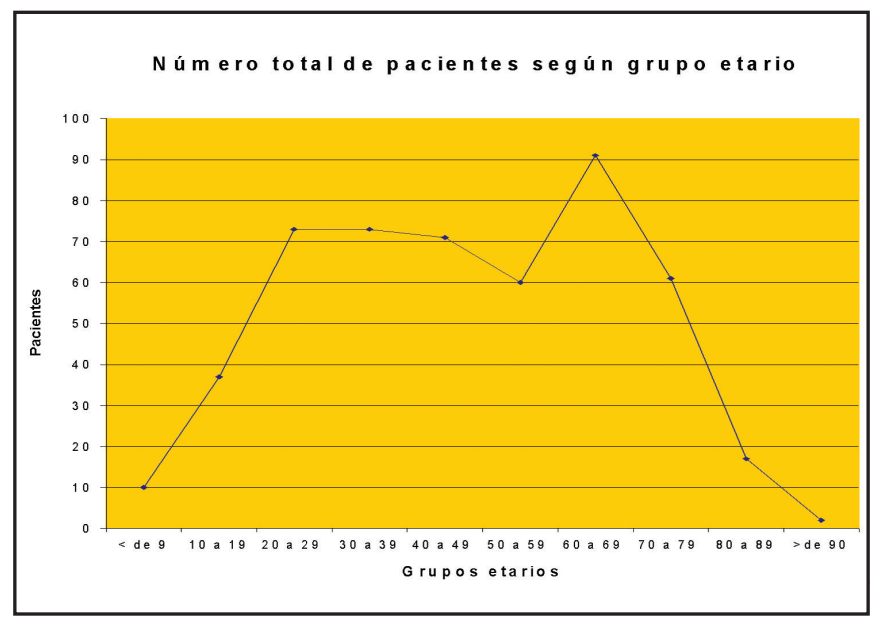

Fig. 1. Número total de pacientes según grupo etario.

\section{Número total de pacientes según sexo}

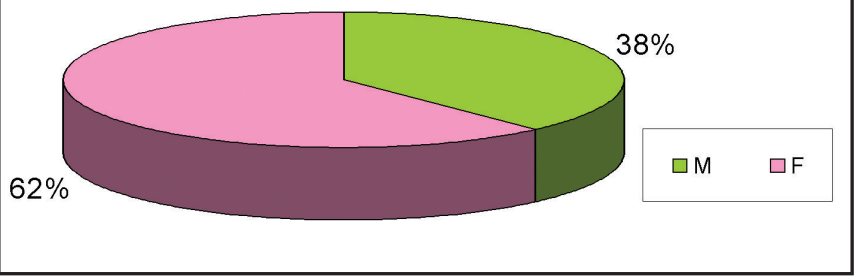

Fig. 2. Número total de pacientes según sexo. 


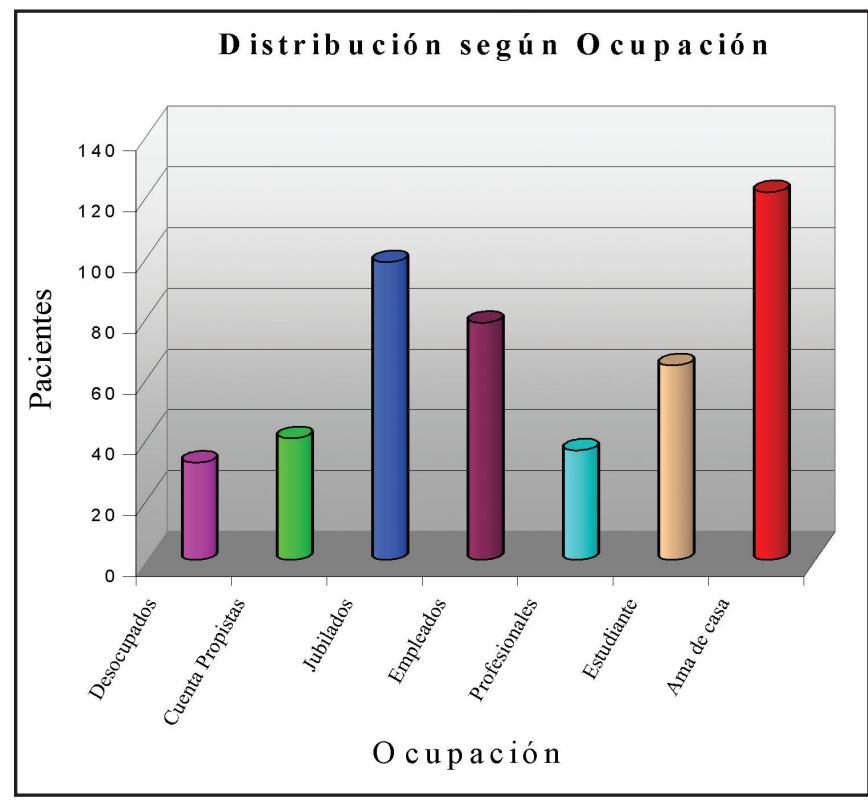

Fig. 3. Distribución según ocupación.

Sobre un total de 549 lesiones y enfermedades registradas se presentó una prevalencia según patologías de:

1. Infecciones de la mucosa bucal: a) Enfermedades bacterianas, 17 (3,1\%); b) Micóticas superficiales y profundas, 108 (19,67\%); c) Virosis, 18 (3,28\%); d) Parasitosis, 2 (0,86\%).

2. Lesiones de enfermedades Inmunológicas, 83 $(15,12 \%)$.

3. Alteraciones de la ATM, $28(5,1)$.

4. Enfermedades de las glándulas salivales, 36 (6,55\%).

5. Patología de las piezas dentarias, $19(3,46)$.

6. Lesiones gingivales y periodontales, $23(4,2 \%)$.

7. Seudotumores de la mucosa bucal $36(6,55 \%)$.

8. Tumores benignos, 22 (4\%).

9. Malformaciones de los tejidos blandos, 4 $(0,73 \%)$.

10. Alteraciones de la pigmentación, 9 (1,64\%).

11. Trastornos bucales de origen neurológico, 15 $(2,73 \%)$.

12. Alteraciones hematológicas, 4 (0,73\%).

13. Lesiones precancerosas, 74 (13,48\%).

14. Tumores malignos, 10 (1,82\%).

15. Enfermedades sistémicas, 35 (6,38\%). Figuras 4 y 5 .

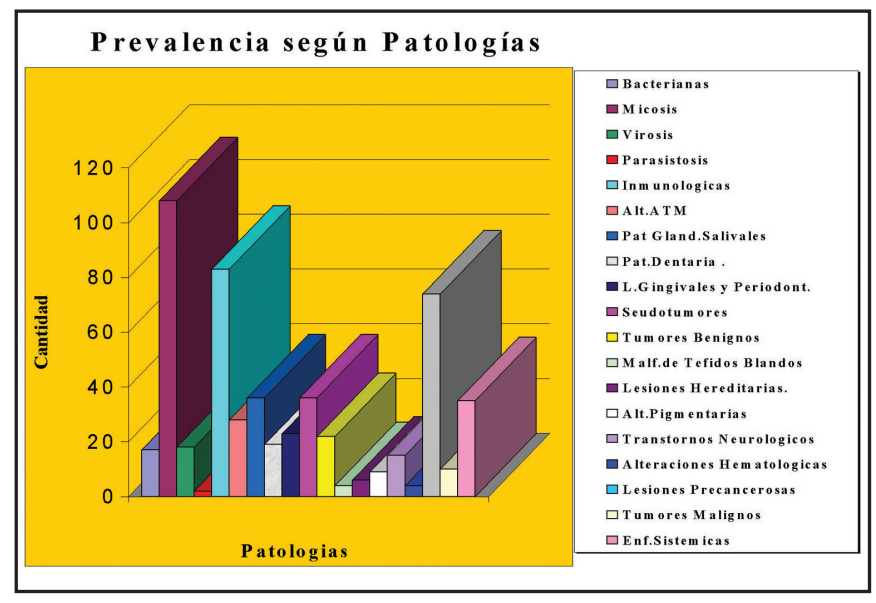

Fig. 4. Prevalencia según grupos de lesiones.

Entre los Métodos Complementarios de Diagnóstico que se utilizaron para llegar a diagnósticos de certeza fueron los más prevalentes la Citología Exfoliativa: 192 (59\%) y la Biopsia: 134 (41\%). Figura 6.

\section{DISCUSIÓN}

Consideramos a este trabajo una investigación de un grupo docente de la Asignatura Patología y Clínica Estomatológica de la Facultad de Odontología de la UNLP.

Habiendo ya establecido en nuestros objetivos las bases del proceso Enseñanza/aprendizaje que tratamos de realizar en la Unidad Hospitalaria a que hacemos referencia y, sobre la base de la falta de datos epidemiológicos sobre la incidencia y la prevalencia de las lesiones, enfermedades y síndromes que afectan el terreno de cara y cuello, y en especial, la cavidad bucal con sus órganos anexos, es que realizamos un relevamiento epidemiológico de las nombradas al mismo tiempo de la función docente hospitalaria.

Podríamos decir que, después de valorar y evaluar las conductas y respuestas de los alumnos a través de todos estos años de nuestra labor, hemos observado que:

1. El contacto directo del alumno con los pacientes ambulatorios e internados ha favorecido el aprendizaje de las diferentes patologías observadas. 
AVANCES EN ODONTOESTOMATOLOGÍA

Vol. 22 - Núm. 5 - 2006

\begin{tabular}{|c|c|c|c|c|c|}
\hline \multicolumn{6}{|l|}{ PATOLOGÍAS } \\
\hline \multirow[t]{4}{*}{ BACTERIANAS } & TBC & $24 \%$ & LESIONES & Hiperplasia gingival & $17 \%$ \\
\hline & Sífilis & $29 \%$ & GINGIVALES & Gingivitis marginal & \\
\hline & Impétigo & $6 \%$ & $\mathrm{Y}$ & Aguda & $30 \%$ \\
\hline & Sinusitis & $41 \%$ & & Crónica & $30 \%$ \\
\hline MICOSIS & & & PERIODONTALES & Periodontitis & $22 \%$ \\
\hline \multirow[t]{4}{*}{ Superficiales } & Candidiasis eritematosa & $41 \%$ & SEUDOTUMORES & Seudopapiloma diapneusico & $36 \%$ \\
\hline & Candidiasis pseudomembranosa & $56 \%$ & & Hiperplasias & $50 \%$ \\
\hline & Candidiasis subplaca & $2 \%$ & & Granuloma piógeno & $3 \%$ \\
\hline & Candidiasis esofágica & $1 \%$ & & Granuloma gravídico & $6 \%$ \\
\hline Profundas & Histoplasmosis & $1 \%$ & & Granuloma gigantocelular periférico & $3 \%$ \\
\hline VIROSIS & & & & Torus & $3 \%$ \\
\hline \multirow[t]{2}{*}{ VHS } & Herpes simple & $33 \%$ & TUMORES & & \\
\hline & Gingivo estomatitis herpética & $22 \%$ & BENIGNOS & Hemangiopericitoma & $5 \%$ \\
\hline \multirow[t]{2}{*}{ HPV } & Condilomas & $22 \%$ & & Papiloma & $23 \%$ \\
\hline & Verrugas vulgares & $6 \%$ & & Hemangioma & $50 \%$ \\
\hline HIV & & $11 \%$ & & Neurinoma & $5 \%$ \\
\hline Paramixovirus & Parotiditis & $5 \%$ & & Lipoma & $9 \%$ \\
\hline PARASITOSIS & & $100 \%$ & & Osteofibroma & $5 \%$ \\
\hline \multirow[t]{6}{*}{ INMUNOLOGICAS } & Aftas & $58 \%$ & & Cementoma & $5 \%$ \\
\hline & Pénfigo vulgar & $12 \%$ & MALFORMACIONES & & \\
\hline & Liquen típico & $22 \%$ & & Puntos de Fordyce & $50 \%$ \\
\hline & Lupus Sistémico & $2 \%$ & & Lengua escrotal & $50 \%$ \\
\hline & Esclerodermia & $1 \%$ & LESIONES & & \\
\hline & Alergias & $5 \%$ & HEREDITARIAS & Nevo esponja blanco & $67 \%$ \\
\hline ALTERACIONES & Síndrome dolor-disfunción & $86 \%$ & & Lengua geográfica & $33 \%$ \\
\hline \multirow[t]{2}{*}{ DE LA ATM } & Latero desviación & $7 \%$ & Alt.PIGMENTARIAS & & \\
\hline & Trismus & $7 \%$ & & Melanosis & $89 \%$ \\
\hline PATOLOGIA DE & & & Vitíligo & $11 \%$ & \\
\hline \multirow[t]{9}{*}{ GLAN.SALIVALES } & Quistes & $50 \%$ & TRANSTORNOS & Glosodignia & $33 \%$ \\
\hline & Malformaciones & & NEUROLOGICOS & Neuralgia & $7 \%$ \\
\hline & Hipertrofia & $11 \%$ & & Síndrome ardor quemazón & $60 \%$ \\
\hline & Atrofia & $8 \%$ & ALTERACIONES & & \\
\hline & Alteraciones funcionales & & HEMATOLOGICAS & Púrpura & $25 \%$ \\
\hline & Hipersialia & $17 \%$ & & Varicosidades & $25 \%$ \\
\hline & Xerostomia & $8 \%$ & & Úlcera eosinófila & $50 \%$ \\
\hline & Inflamatorias & & LESIONES & & \\
\hline & Sialodoquitis & $6 \%$ & PRECANCEROSAS & Leucoplasia36\% & \\
\hline PATOLOGÍA & & & & Liquen atípico & $31 \%$ \\
\hline \multirow[t]{4}{*}{ DENTARIA } & Pulpitis & $11 \%$ & & Eritroplasia & $1 \%$ \\
\hline & Proceso periapical & $32 \%$ & & Úlcera traumática & $31 \%$ \\
\hline & Fístula & $47 \%$ & TUMORES & & \\
\hline & Diente retenido & $11 \%$ & MALIGNOS & Ca. de células escamosas & $70 \%$ \\
\hline ENFERMEDADES & Bulimia & $3 \%$ & & Linfoma No Hodking & $10 \%$ \\
\hline \multirow[t]{5}{*}{ SISTEMICAS } & Hipotiroidismo & $9 \%$ & & Ca. Baso celular & $10 \%$ \\
\hline & Diabetes & $11 \%$ & & Granuloma letal de la línea media & $10 \%$ \\
\hline & Hipertensión & $69 \%$ & & & \\
\hline & Colesterolemia & $6 \%$ & & & \\
\hline & Cardiopatías & $6 \%$ & & & \\
\hline
\end{tabular}

Fig. 5. Distribución según lesiones y enfermedades. 


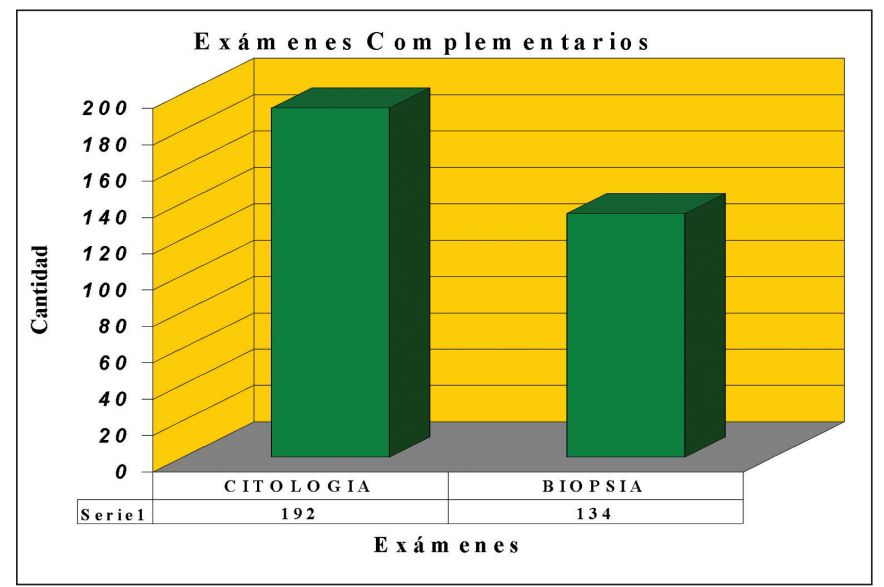

Fig. 6. Exámenes complementarios.

2. Que tal contacto ha estimulado el interés para poder adoptar las conductas necesarias y poder arribar a la correcta resolución de los problemas bajo la guía docente.

3. Que la concurrencia al Hospital les ha permitido a los alumnos ver de cerca las patologías bucales.

4. Que han observado y practicado la integración con los otros servicios médicos afines a la estomatología. Solamente aquellas facultades de países económicamente desarrollados tienen el privilegio de contar con un Hospital Universitario anexo a la cátedra y en donde los alumnos puedan ejercer sus prácticas hospitalarias.

\section{CONCLUSIONES}

1. Creemos que, dados los resultados, se justifica en nuestro medio la inserción de alumnos de Odontología, en especial aquellos que cursan $\mathrm{Pa}$ tología y Clínica Estomatológica, en más de un medio hospitalario. Surge la necesidad que puedan concurrir más cantidad de alumnos a mayor número de hospitales y que cada uno tenga la posibilidad de visitas más numerosas.

2. La distribución por grupos etarios nos mostró una mayor incidencia y prevalencia de todas las lesiones y enfermedades entre los 60 y 69 años de edad.

3. La relación entre sexo masculino y femenino fue de 189 lesiones (38\%) para los primeros con preponderancia de 306 lesiones (62\%) para las segundas.

4. Las ocupaciones de amas de casa y jubilados fueron las más prevalentes, $121(25,8 \%)$ y 98 (20,9\%), respectivamente.

5. Las Micosis superficiales, entre ellas la Candidiasis de la mucosa bucal fue la más prevalente (Candidiasis eritematosa 41\%, Candidiasis Pseudomembranosa 56\%) y de las enfermedades a considerar, las referentes al Sistema Inmunológico se destacaron por su alta incidencia y prevalencia (Aftas 58\%, Líquenes típicos 22\%, Pénfigo vulgar $12 \%)$.

6. El cáncer bucal sigue siendo un problema dentro de nuestra comunidad.

\section{BIBLIOGRAFÍA}

1. ACME-TRI report: Educating Medical Students: Assesing change in medical education. Academic Medicine. Volume 68.Number 6 supplement. June 1993.

2. Carretero, M(comp.): Procesos de enseñanza y aprendizaje. Aique.Bs.As. 1998.

3. Galli A, de Hernández NC, Galperín J: Una Técnica no convencional para la evaluación del aprendizaje: el coloquio. En Temas Universitarios. Revista de Planificación Universitaria No 5. Barquisimeto. Venezuela. Junio 1978.

\section{CORRESPONDENCIA}

\section{Z Casariego}

Bartolomé Mitre 1371-4m-

Buenos Aires. Argentina.

T/FAX: 0054-11-4-3720444

Patología@folp.unlp.edu.ar 